\title{
AN IMPRIMITIVITY THEOREM FOR REPRESENTATIONS OF LOCALLY COMPACT GROUPS ON ARBITRARY BANACH SPACES
}

\author{
Niels GRønBæK
}

\begin{abstract}
We prove a general version of Mackey's Imprimitivity Theorem for induced representations of locally compact groups. Let $G$ be a locally compact group and let $H$ be a closed subgroup. Following Rieffel we show, using Morita equivalence of Banach algebras, that systems of imprimitivity for induction from strongly continuous Banach $H$-modules to strongly continuous Banach $G$-modules can be described in terms of an action on the induced module of $C_{0}(G / H)$, the algebra of complex continuous functions on $G / H$ vanishing at $\infty$, which is compatible with the $G$-homogeneous structure of $G / H$ and the strong operator topology continuity of the module action of $G$.
\end{abstract}

\section{Introduction.}

The notion of induced representations goes back to Frobenius [Fro] and has been a fundamental concept in the development of representation theory. The key question is how well the representation theory of a group is determined by that of its subgroups. In the early 50's Mackey [M1, M2, M3] and others worked on this in the case of unitary representations of a locally compact group, see [M4, Appendix] for an account of this development. Let $G$ be a locally compact group and let $H$ be a closed subgroup. Loosely speaking, inducing representations from $H$ to $G$ is a functorial way of assigning to each unitary representation of $H$ a unitary representation of $G$. The two basic problems are, firstly: how does one recognize a unitary representation of $G$ to be induced from $H$ ? and secondly, which unitary representations of $G$ can be synthesized from induced representation?

In this paper we are concerned with the problem of recognition. In the paper [M1] Mackey characterized induced representations by means of socalled systems of imprimitivity, that is, a measure whose values are projections operating on the representation space in such a way that the action is compatible with the structure of the $G$-homogeneous space $G / H$.

In dealing with unitary representation a natural framework is that of operator algebras. (Indeed, representation theory for locally compact groups 
was a main motivation for the formation of the theory of operator algebras and the two subjects have drawn fruitfully upon each other, see for instance [M4, Appendix, Section 1].) This was further developed by J.G.M. Fell, M.A. Rieffel and others leading to a theory for induced representations of operator algebras and, more generally, Banach*-algebraic bundles, see the introduction to [Fell]. Building on his own notion of Morita equivalence, Rieffel [R2] obtained a simplified and more transparent proof of Mackey's Imprimitivity Theorem.

Unitary representations being of fundamental importance, there are nevertheless several reasons for including Banach space representations or even representations on more general topological vector spaces, see [M4], Section 8. In [Rig] a very general notion of induced representations is studied, and our approach can be seen as a more restrictive generalization than that of [Rig]. In fact, as in [Rig, Section 8], our (strictly) induced modules, when induced from unitary representations of closed subgroups, is easily seen to be Naimark equivalent to Mackey's induced module. However, there are also good reasons for studying Banach space representations apart from their connections with unitary representations. For instance, the theory of groups of automorphisms and the theory of transference couples can naturally be treated as representation theories on Banach spaces. A third example comes from topology, where the study of bounded representations of the fundamental group (in its discrete topology) is of much current interest [Gri].

In the papers [G1] and [G2] we have constructed a theory of Morita equivalence for Banach algebras. In [G2] we proved (Theorem II.17) that an induction functor is - under the natural conditions of having a right adjoint - given by tensoring, thereby making an extension of Mackey's Imprimitivity Theorem accessible through the approach of Rieffel in the general case when the group is acting on an arbitrary Banach space. In the main result of this paper we characterize induced representations by means of systems of imprimitivity completely analogous to those of Rieffel for unitary representations. On the route we show that $L_{1}(G)$, viewed as a Banach right module over the group algebra $L_{1}(H)$ by means of convolution of measures, is a (strictly) flat generator of the category of essential right Banach modules over $L_{1}(H)$, a result that perhaps is interesting in itself. Our method of proof is, except for a technical complication of choosing measurable cross sections $([\mathbf{K e h}])$, elementary, building only on basic properties of integration on homogeneous space.

We finish the paper by illustrating the results on the case $G=\mathbb{R}$ and $H=\mathbb{Z}$. It turns out that induced representations can be characterized by having infinitesimal generators, which are obtained by lifting differentiation on the circle group $\mathbb{R} / \mathbb{Z}$. 


\section{Preliminaries.}

In this chapter we introduce some notation along with the basic setting in which we are working. We adhere to the notation and terminology of [G1, G2]. For a Banach algebra $\mathfrak{A}$ we consider its category of left Banach $\mathfrak{A}$-modules and bounded module morphisms, denoted $\mathfrak{A}-\mathbf{m o d}$, and the full subcategory ind $\mathfrak{A}-\bmod$ whose objects $X \in$ ind $\mathfrak{A}-\bmod$ satisfy $X \cong \mathfrak{A} \widehat{\widehat{Q}} X$ canonically. If $\mathfrak{A}$ has a bounded left approximate identity, then $X \in$ ind $\mathfrak{A}-\mathbf{m o d}$ if and only if $X$ is essential, that is, $X=(\mathfrak{A} . X)^{-}$. The Banach algebra $\mathfrak{A}$ is called self-induced if $\mathfrak{A} \in$ ind $\mathfrak{A}-\mathbf{m o d}$. We also consider the analogous right and two-sided concepts. We use the notation $\mathfrak{A} \mathbf{h}, \mathbf{h}_{\mathfrak{A}}, \mathfrak{A} \mathbf{h}_{\mathfrak{A}}$, etc. for the spaces of morphisms. The category of Banach spaces and bounded linear maps is denoted Ban. The Banach algebra of bounded operators on a Banach space $X$ is denoted $\mathcal{B}(X)$. If $G$ is a locally compact group, we denote the category of left Banach $G$-modules with bounded strongly continuous $G$-action and bounded module morphisms by $G$-mod. Choosing a fixed Haar measure $\lambda$ on $G$ it can easily be shown that $G-\mathbf{m o d}$ can be identified with ind $-L_{1}(G)-\bmod$ through

$$
f \cdot \xi=\int_{G} f(g) g \cdot \xi d \lambda(g) \quad\left(f \in L_{1}(G), \xi \in X \in G \text {-mod }\right) .
$$

With this identification (and its right and two-sided analogues) we also get an identification of the tensor products $\underset{G}{\widehat{\otimes}}$ and $\underset{L_{1}(G)}{\widehat{\otimes}}$, see for instance [R1, Theorem 3.14].

Let $\mathfrak{A}$ be a self-induced Banach algebra and let $P \in$ ind mod-a. The module $\mathfrak{A} \widehat{\otimes} \mathbf{h}_{\mathfrak{A}}(P, \mathfrak{A}) \in$ ind $\mathfrak{A}-\mathbf{m o d}$ is denoted $P^{\sharp}$. We form the Banach algebra $\mathcal{E} \stackrel{\mathfrak{A}}{=} P \widehat{\otimes} \widehat{\mathbb{A}} P^{\sharp}$ with product given by

$$
(x \underset{\mathfrak{A}}{\otimes} \varphi)(y \underset{\mathfrak{A}}{\otimes} \psi)=x \varphi(y) \underset{\mathfrak{A}}{\otimes} \psi \quad\left(x, y \in P ; \varphi, \psi \in P^{\sharp}\right) .
$$

Let $(\cdot, \cdot): P^{\sharp} \times P: \rightarrow \mathfrak{A}$ and $[\cdot, \cdot]: P \times P^{\sharp} \rightarrow \mathcal{E}$ be the natural balanced pairings. We call the tuple $\left(\mathfrak{A}, \mathcal{E}, P, P^{\sharp},(\cdot, \cdot),[\cdot, \cdot]\right)$ the Morita-context derived from $P$. If $\mathcal{E}$ is self-induced, and if the pairing $(\cdot, \cdot)$ gives rise to an isomorphism $P^{\sharp} \widehat{\mathcal{E}} P \cong \mathfrak{A}$, we will say that $\mathfrak{A}$ and $\mathcal{E}$ are Morita equivalent, denoted $\mathcal{E} \approx \mathfrak{A}$. A sufficient condition that a given Morita-context gives Morita equivalence between self-induced Banach algebras is that $P_{\mathfrak{A}}$ is a flat generator of ind $\bmod -\mathfrak{A}$, see [G2, Theorem IV.6].

Let $\mathfrak{A}$ and $\mathcal{B}$ be Banach algebras and let $P \in \mathfrak{A}-\mathbf{m o d}-\mathcal{B}$. The functor $\mathcal{B}-\bmod \rightarrow \mathfrak{A}-\bmod$ given by $X \rightarrow P \widehat{\widehat{Q}} X$ is called an induction functor, see also [G2, Definition II.18]. 
We finish this section with some notational conventions. For a locally compact space $K$ the symbols $C_{c}(K), C_{l u}(K), C_{r u}(K), C_{u}(K)$, or $C_{b}(K)$ denote the spaces of complex continuous functions which are respectively compactly supported, (left-, right- ) uniformly continuous, or bounded. If $X$ is a Banach space with dual space $X^{*}$, then pointed brackets $\langle\cdot, \cdot\rangle$ always refer to the standard duality $\langle\cdot, \cdot\rangle: X \times X^{*} \rightarrow \mathbb{C}$. In particular if $(\Omega, \Sigma, \nu)$ is a measure space, then $\langle\cdot, \cdot\rangle: L_{1}(\Omega, \nu) \times L_{\infty}(\Omega, \nu) \rightarrow \mathbb{C}$. is given by $\langle f, m\rangle=\int_{\Omega} f(u) m(u) d \nu(u) \quad\left(f \in L_{1}(\Omega, \nu), m \in L_{\infty}(\Omega, \nu)\right)$. If $H$ is a closed subgroup of a locally compact group $G$, then $\lambda$ will denote (a fixed) Haar measure on $G$ and $\mu$ will denote (a fixed) Haar measure on $H$. The corresponding quasi-invariant measure on $G / H$ will be denoted $\dot{\lambda}$. The modular functions on $G$ and $H$ are denoted $\Delta$ and $\delta$ respectively.

\section{An abstract imprimitivity theorem à la Rieffel.}

We start by summarizing the basic idea behind Rieffel's proof of Mackey's Imprimitivity Theorem [R2]. We build on the approach in [R4] where the idea of the construction is summarized in a purely algebraic setting.

Suppose we have two Banach algebras $\mathfrak{A}$ and $\mathcal{B}$ and an induction functor $\mathcal{B}-\bmod \rightarrow \mathfrak{A}-\mathbf{m o d}$, given by tensoring with a module ${ }_{\mathfrak{A}} P_{\mathcal{B}}$. The "imprimitivity" questions is: How does one recognize a module in $\mathfrak{A}-\mathbf{m o d}$

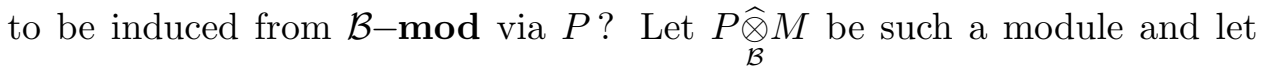
$\left(\mathcal{B}, \mathcal{E}, P, P^{\sharp},(),,[],\right)$ be the Morita context derived from $P_{\mathcal{B}}$. Then $P$ is naturally in $\mathcal{E}$ - $\bmod$ and $P \widehat{\bigotimes}_{\mathcal{B}} M$ inherits an $\mathcal{E}$-module structure from ${ }_{\mathcal{E}} P$. Likewise $\mathcal{E}$ inherits an $\mathfrak{A}$-module structure from ${ }_{\mathfrak{A}} P$. These module structures are related by the associative law

$$
\text { a.(e.p) }=(\text { a.e).p } \quad(a \in \mathfrak{A}, e \in \mathcal{E}, p \in P) .
$$

This is Rieffel's criterion for an abstract system of imprimitivity, see also [R2]. Note that (II.1) can naturally be extended to hold when $e$ is replaced by any endomorphism in $\mathbf{h}_{\mathcal{B}}(P, P)$.

Of course $P \widehat{\otimes_{\mathcal{B}}} M$ is just one way of completing the algebraic tensor product $P \underset{\mathcal{B}}{\otimes} M$ to a module in $\mathfrak{A}$-mod. In order to describe a more general situation we first make a definition.

Definition II.1. Let $X \in \mathfrak{A}-\mathbf{m o d}$. We say that $X$ is essentially induced from $\mathcal{B}-\mathbf{m o d}$ via ${ }_{\mathfrak{A}} P_{\mathcal{B}}$ if there is $M \in \mathcal{B}-\mathbf{m o d}$ and $i \in \mathfrak{a}_{\mathfrak{h}} \mathbf{h}\left(P \underset{\mathcal{B}}{\otimes} \widehat{\otimes}_{1} M\right)$, such that for each $T \in \mathbf{h}_{\mathcal{B}}(P, P)$ there is a unique $\widetilde{T} \in \mathcal{B}(X)$ making the diagram 


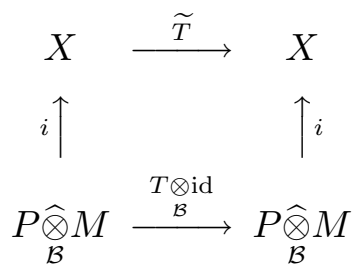

commutative. If $i$ can be chosen as an injective map we say that $X$ is faithfully induced and if $i$ can be chosen an isomorphism we call $X$ strictly induced.

Example II.2. The injective tensor product $P \underset{\mathcal{B}}{\otimes} M$ is essentially, but in general not strictly, induced via $P$. If $P_{\mathbb{C}}$ has the approximation property then $P \underset{\mathbb{C}}{\otimes} M$ is faithfully induced. If $P_{\mathbb{C}}$ fails the approximation property we may choose $M$ so that $P \underset{\mathbb{C}}{\otimes} M$ is not faithfully induced via $P$.

We now formulate the imprimitivity principle.

Theorem II.3. Let $\mathfrak{A}$ and $\mathcal{B}$ be Banach algebras with $\mathcal{B}$ self-induced. Let $P \in \mathfrak{A}-\mathbf{m o d}-\mathcal{B}$ such that $P_{\mathcal{B}} \in$ ind $\mathbf{m o d}-\mathcal{B}$ and such that the Morita context derived from $P_{\mathcal{B}}$ gives a self-induced Banach algebra $\mathcal{E}$ with $\mathcal{E} \approx \mathcal{B}$. Consider $X \in \mathfrak{A}$-mod. Then $X$ is essentially induced from ind $\mathcal{B}$ - $\bmod$ via $P_{\mathcal{B}}$, if and only if there is a Banach module action of $\mathcal{E}$ on $X$ and there are $Y \in$ ind $\mathcal{E}$-mod and $j \in{ }_{\mathcal{E}} \mathbf{h}(Y, X)$ such that for each $T \in \mathbf{h}_{\mathcal{B}}(P, P)$ there is a unique $\widetilde{T}: X \rightarrow X$ making the diagram

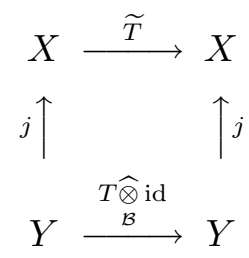

commutative and such that $\widetilde{L_{a}}=L_{a}$ for all $a \in \mathfrak{A}$, where $L_{a}$ is the generic notation for left module multiplication by $a \in \mathfrak{A}$.

$X$ is faithfully induced, if and only if in addition $j$ can be chosen injective and $X$ is strictly induced if and only if $X \in \operatorname{ind} \mathcal{E}$-mod. In the latter case we only need to check commutativity of the diagrams for $T=L_{a}(a \in \mathfrak{A})$.

Proof. This is (almost) a paraphrasing of the definitions involved. First note that since $Y \cong P \widehat{\mathcal{B}} \widehat{Q}^{\sharp} \widehat{\otimes}_{\mathcal{E}} Y$ we have $T \widehat{\otimes}_{\mathcal{B}}$ id $\in \mathcal{B}(Y)$, so that the diagram makes sense. Suppose that $X$ is essentially induced by means of $i: P \widehat{\mathcal{B}} M \rightarrow X$. 
Put $Y=P \widehat{\bigotimes}_{\mathcal{B}} M$ and $j=i$. It follows from the closed graph theorem that $X$ is a Banach module over $\mathbf{h}_{\mathcal{B}}(P, P)$. Furthermore, from the commutativity of the diagrams (II.2) we see that $i$ is also an $\mathbf{h}_{\mathcal{B}}(P, P)$-module homomorphism, when $Y$ has its canonical $\mathbf{h}_{\mathcal{B}}(P, P)$-module structure. Since $i$ is an $\mathfrak{A}$-module homomorphism it follows from uniqueness that $\widetilde{L_{a}}=L_{a}$.

Conversely, suppose $X$ satisfies the conditions. Put $M=P^{\sharp} \widehat{\mathcal{E}} Y$. Then $M \in$ ind $\mathcal{B}$-mod and $P \widehat{\mathcal{B}} M$ is isomorphic to $Y$ as $\mathcal{E}$-modules, since $\mathcal{E} \approx \mathcal{B}$. The commutativity of the diagrams (II.3) shows that $\underset{\mathcal{B}}{\widehat{\otimes}} M \stackrel{\cong}{\rightrightarrows} Y \stackrel{j}{\rightarrow} X$ is also an $\mathfrak{A}$-module homomorphism. In other words, modulo similarity, the maps $i$ of the definition of essentially induced modules and $j$ in the statement of the theorem, are identical. The rest is clear.

Remark. Corresponding to faithful inducing there is also a notion of fully induced modules. Since these will merely be quotients of $P \widehat{\otimes_{\mathcal{B}}} M$, they appear less interesting. It can easily be seen that, if $X$ is fully induced from $M$ via $P$, then $X$ is faithfully induced from $M / K$, where $K$ is the kernel of the map $M \cong P^{\sharp} \underset{\mathcal{E}}{\widehat{\otimes}} P \widehat{\mathcal{B}} M \rightarrow P^{\sharp} \underset{\mathcal{E}}{\widehat{\otimes}} X$.

\section{The module $L_{1}(G)_{H}$.}

Our aim is to apply the abstract imprimitivity theorem in the case $\mathfrak{A}=$ $L_{1}(G)$ and $\mathcal{B}=L_{1}(H)$, where $G$ is a locally compact group and $H$ is a closed subgroup of $G$. As inducing module we shall consider $L_{1}(G)_{H}$ where the action of $L_{1}(H)$ is given by convolution of measures, viewing Haar measures on $H$ as Borel measures on $G$. In the case where $H$ is open in $G$ the induction functor is the left adjoint of the restriction functor $G-\bmod \rightarrow H-\bmod$, cf. [R1, Theorem 5.1] and [G2, Theorem II.17].

Since $L_{1}(H)$ has a bounded approximate identity a module $P_{H}$ is an equivalence module to its derived algebra if and only if it is a generator and its derived algebra will be an $\mathrm{H}$-unital Banach algebra ([Wod]), if $P_{H}$ is flat, see [G2, Theorem IV.6]. So our first goal is to investigate the module $L_{1}(G)_{H}$. We start by showing that it is a flat $L_{1}(H)$-module:

Theorem III.1. Let $G$ be a locally compact group and let $H$ be a closed subgroup. Then the module $L_{1}(G)_{H}$ is flat in $\mathbf{m o d}-H$.

Proof. Let

$$
0 \rightarrow X \stackrel{j}{\rightarrow} Y \rightarrow Z \rightarrow 0
$$


be a short exact sequence of modules in $H$-mod. We must show that

$$
0 \rightarrow L_{1}(G) \underset{H}{\widehat{\otimes}} X \rightarrow L_{1}(G) \underset{H}{\widehat{\otimes}} Y \rightarrow L_{1}(G) \underset{H}{\widehat{\otimes}} Z \rightarrow 0
$$

is short exact in Ban. Dualizing, we must show that

$$
0 \rightarrow \mathbf{h}_{H}\left(L_{1}(G), Z^{*}\right) \rightarrow \mathbf{h}_{H}\left(L_{1}(G), Y^{*}\right) \rightarrow \mathbf{h}_{H}\left(L_{1}(G), X^{*}\right) \rightarrow 0
$$

is short exact in Ban, that is, we must show that, if $\varphi \in \mathbf{h}_{H}\left(L_{1}(G), X^{*}\right)$ then there is $\tilde{\varphi} \in \mathbf{h}_{H}\left(L_{1}(G), Y^{*}\right)$ making the diagram

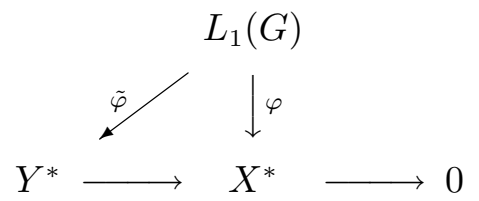

commutative.

Now let $f \in L_{1}(G)$ and let $\left(e_{i}\right)_{i \in I}$ be a bounded approximate identity for $L_{1}(G)$. Then we have the formula

$$
\left\langle x, \varphi\left(e_{i} f\right)\right\rangle=\int_{G} f(u)\left\langle x, \varphi\left(e_{i} . u\right)\right\rangle d \lambda(u) \quad(x \in X) .
$$

Let $\beta: G \rightarrow \mathbb{R}_{+} \cup\{0\}$ be a Brûhat approximate cross section with respect to $H$ (see [Bour, Proposition 8, p. 51]), and define $\varphi_{i}: M(G) \rightarrow X^{*}$ by

$$
\varphi_{i}(\mu)=\varphi\left(e_{i} \mu\right) \quad(\mu \in M(G)) .
$$

Then $\varphi_{i}$ is continuous with respect to the s.o. topology on $M(G)$. In the following we identify $u \in G$ with the point mass at $u$ and shall write $\varphi_{i}(u)$ for $\varphi_{i}$ evaluated at the point mass at $u$. We have for $x \in X, f \in L_{1}(G)$

$$
\begin{aligned}
\left\langle x, \varphi_{i}(f)\right\rangle & =\int_{G} f(u)\left\langle x, \varphi_{i}(u)\right\rangle d \lambda(u) \\
& =\int_{G} \int_{H} f(u)\left\langle x, \varphi_{i}(u)\right\rangle \beta\left(u t^{-1}\right) \delta\left(t^{-1}\right) d \mu(t) d \lambda(u) \\
& =\int_{H} \int_{G} f(u t)\left\langle x, \varphi_{i}(u t)\right\rangle \beta(u) \Delta(t) \delta\left(t^{-1}\right) d \lambda(u) d \mu(t) \\
& =\int_{G} \int_{H} f(u t)\left\langle t . x, \varphi_{i}(u)\right\rangle \beta(u) \Delta(t) \delta\left(t^{-1}\right) d \mu(t) d \lambda(u) .
\end{aligned}
$$

Now let $\varepsilon>0$, and let $\mathfrak{E} \subseteq X$ and $\mathfrak{F} \subseteq L_{1}(G)$ be finite sets. Choose a measurable step function $\sigma: G \rightarrow X^{*}$ of the form

$$
\sigma=\sum_{k} \varphi_{i}\left(u_{k}\right) \chi_{E_{k}}
$$


with $E_{k}$ 's mutually disjoint such that

$$
\left|\left\langle x, \sigma f-\varphi_{i}(f)\right\rangle\right|<\varepsilon \quad(x \in \mathfrak{E}, f \in \mathfrak{F}),
$$

with $\sigma f$ denoting pointwise multiplication. This is clearly possible, since the function $u \rightarrow\left\langle x, \varphi_{i}(u)\right\rangle$ is continuous and bounded. Consider the map $j: X \rightarrow Y$ from (III.1). By the open mapping theorem we may choose $y_{k}^{*} \in Y^{*}$ such that $j^{*}\left(y_{k}^{*}\right)=\varphi_{i}\left(u_{k}\right)$ and $\sup \left\|y_{k}^{*}\right\|=C<\infty$.

Let $\Gamma=I \times \mathcal{P}_{e}(X) \times \mathcal{P}_{e}\left(L_{1}(G)\right) \times \mathbb{R}_{+}$where $\mathcal{P}_{e}(\cdot)$ denote the set of finite subsets. Define for $\gamma=(i, \mathfrak{E}, \mathfrak{F}, \varepsilon) \in \Gamma$ a function $\tilde{\varphi}_{\gamma}: L_{1}(G) \rightarrow Y^{*}$ given by

$$
\tilde{\varphi}_{\gamma}(f)=\int_{G} \int_{H} \sum_{k} f(u t) \chi_{E_{k}}(u) \beta(u) \Delta(t) \delta\left(t^{-1}\right) y_{k}^{*} \cdot t d \mu(t) d \lambda(u)
$$

where the $y_{k}^{*}$ 's are chosen in accordance with (III.4). We want to prove that the integral exists as a Bochner integral. First of all, the integrand is measurable, since we may assume that $t \rightarrow y_{k}^{*}$.t is continuous, $j^{*}\left(y_{k}^{*}\right)$ being in the range of $\varphi_{i}$. Since the $E_{k}$ 's are disjoint we have

$$
\begin{aligned}
& \int_{H} \int_{G} \sum_{k}|f(u t)| \chi_{E_{k}}(u) \beta(u) \Delta(t) \delta\left(t^{-1}\right)\left\|y_{k}^{*} \cdot t\right\| d \lambda(u) d \mu(t) \\
& \leq C \int_{H} \int_{G} \sum_{k}|f(u)| \chi_{E_{k}}\left(u t^{-1}\right) \beta\left(u t^{-1}\right) \delta\left(t^{-1}\right) d \lambda(u) d \mu(t) \\
& =C \int_{G}|f(u)| \int_{H} \sum_{k} \chi_{E_{k}}(u t) \beta(u t) d \mu(t) d \lambda(u) \\
& \leq C \int_{G}|f(u)| \int_{H} \beta(u t) d \mu(t) d \lambda(u)=C\|f\| .
\end{aligned}
$$

Hence the linear maps $\tilde{\varphi}_{\gamma}$ form a bounded net of bounded operators $\left\|\tilde{\varphi}_{\gamma}\right\| \leq$ C.

If we order $\Gamma$ suitably we have by the estimate (III.4) that $\lim _{\gamma} j^{*} \circ \tilde{\varphi}_{\gamma}=\varphi$ in the $\mathrm{w}^{*}$-operator topology. Identifying $\mathcal{B}\left(L_{1}(G), Y^{*}\right)$ with $\left(L_{1}(G) \widehat{\otimes} Y\right)^{*}$ we note that the $\mathrm{w}^{*}$-operator topology on $\mathcal{B}\left(L_{1}(G), Y^{*}\right)$ is a $\mathrm{w}^{*}$-topology. By $\mathrm{w}^{*}$-compactness we may thus assume that a suitable subnet of $\left(\tilde{\varphi}_{\gamma}\right)_{\Gamma}$ is $\mathrm{w}^{*}$ operator convergent to a bounded linear map $\tilde{\varphi}: L_{1}(G) \rightarrow Y^{*}$. Since $j^{*}$ is $\mathrm{w}^{*}-\mathrm{w}^{*}$ continuous we have $j^{*} \circ \tilde{\varphi}=\varphi$.

It remains to show that $\tilde{\varphi}$ is an $L_{1}(H)$-homomorphism. For this, it 
suffices to show that $\tilde{\varphi}_{\gamma}$ is. But for $f \in L_{1}(G), t \in H, y \in Y$ we have

$$
\begin{aligned}
& \left\langle y, \tilde{\varphi}_{\gamma}(f . t)\right\rangle \\
& =\int_{G} \int_{H} \sum_{k} f\left(u s t^{-1}\right) \Delta\left(t^{-1}\right) \chi_{E_{k}}(u) \beta(u) \Delta(s) \delta\left(s^{-1}\right)\left\langle y, y_{k}^{*} . s\right\rangle d \mu(s) d \lambda(u) \\
& =\int_{G} \int_{H} \sum_{k} f(u s) \chi_{E_{k}}(u) \beta(u) \Delta(s) \delta\left(s^{-1}\right)\left\langle t . y, y_{k}^{*} . s\right\rangle d \mu(s) d \lambda(u) \\
& =\left\langle t . y, \varphi_{\gamma}(f)\right\rangle .
\end{aligned}
$$

This finishes the proof that $L_{1}(G)_{H}$ is flat as a $H$-module.

We now continue to show that $L_{1}(G)_{H}$ is a generator of $\bmod -H$. It suffices to show that $L_{1}(G)_{H}$ generates $L_{1}(H)_{H}$. In order to do so we shall need the following technical result.

Lemma III.2. Let $G$ and $H$ be as above and let $V$ be a neighborhood of e in $H$. Then there is a function $f \in L_{1}(G)$ and $T \in \mathbf{h}_{H}\left(L_{1}(G), L_{1}(H)\right)$ such that

(a) $T f$ is continuous on $H$

(b) $\|T\|=\|f\|=1$

(c) $\int_{H} T f(t) d \mu(t)=1$

(d) Tf vanishes outside $V$.

Proof. Let $x \rightarrow \dot{x}$ denote the natural map $G \rightarrow G / H$ and let $\dot{\lambda}$ be the quasi invariant measure on $G / H$ corresponding to $\lambda$. Then [Bour, Theorem 2, p. 56] there is a continuous function $\rho: G \rightarrow(0, \infty)$ such that

$$
\rho(x s)=\frac{\delta(s)}{\Delta(s)} \rho(x) \quad(x \in G, s \in H)
$$

and such that for each $f \in L_{1}(G)$ we have

$$
\int_{G} f(x) d \lambda(x)=\int_{G / H} \frac{1}{\rho(x)} \int_{H} \frac{\Delta(s)}{\delta(s)} f(x s) d \mu(s) d \dot{\lambda}(\dot{x}),
$$

where the integrand on the right is constant on cosets, is defined $\dot{\lambda}$-almost everywhere, and is measurable outside a set of $\dot{\lambda}$-measure 0 . Since both $\mu$ and $\dot{\lambda}$ are completion regular [Bour] we may choose a compact $G_{\delta^{-}}$set, $W \subseteq H$ such that $W^{-1} W \subseteq V$ and $0<\mu(W)<\infty$ and a Baire set $\mathcal{C} \subseteq G / H$ such that $\dot{\lambda}(\mathcal{C})=1$. By the main result of $[\mathbf{K e h}]$ there is a set $C \subseteq G$ such that

(i) $C$ is relatively compact. 
(ii) $C \cap(x H)$ contains at most one point for each $x \in G$.

(iii) $\dot{C}=\mathcal{C}$.

(iv) $C W$ is a Baire set.

Let $\kappa$ be the characteristic function $\chi_{C W}$ of the set $C W$. Then $\kappa \in L_{1}(G) \cap$ $L_{\infty}(G)$. We look at

$$
\|\kappa\|_{1}=\int_{G / H} \frac{1}{\rho(x)} \int_{H} \chi_{C W}(x s) \frac{\Delta(s)}{\delta(s)} d \mu(s) d \dot{\lambda}(\dot{x}) .
$$

Since the integrand is constant on cosets we get the estimate

$$
\|\kappa\|_{1} \leq \dot{\lambda}(\mathcal{C}) \sup \left\{\frac{1}{\rho(c)} \mid c \in C\right\} \mu(W) \sup \left\{\frac{\Delta(s)}{\delta(s)} \mid s \in W\right\} .
$$

Since $\dot{\lambda}(\mathcal{C})=1$, since $C$ is relatively compact, and since $\rho, \Delta, \delta$ are continuous this estimate is finite. In a similar way we get $\|\kappa\|_{1}>0$.

Define $T_{\kappa}: L_{1}(G) \rightarrow L_{1}(H)$ by

$$
\left(T_{\kappa} f\right)(t)=\left\langle f . t^{-1}, \kappa\right\rangle \delta\left(t^{-1}\right) \quad(t \in H) .
$$

Then $T_{\kappa} f$ is continuous. We estimate its norm

$$
\begin{aligned}
& \int_{H}\left|\left(T_{\kappa} f\right)(t)\right| d \mu(t) \\
& =\int_{H} \delta\left(t^{-1}\right)\left|\int_{G} f(x t) \Delta(t) \kappa(x) d \lambda(x)\right| d \mu(t) \\
& \leq \int_{G} \int_{H} \delta\left(t^{-1}\right)|f(x t)| \Delta(t) \kappa(x) d \mu(t) d \lambda(x) \\
& =\int_{G / H} \frac{1}{\rho(x)} \int_{H} \kappa(x s) \int_{H}|f(x s t)| \frac{\Delta(t)}{\delta(t)} d \mu(t) \frac{\Delta(s)}{\delta(s)} d \mu(s) d \dot{\lambda}(\dot{x}) \\
& =\int_{G / H} \frac{1}{\rho(x)} \int_{H} \kappa(x s) \int_{H} f(x s t) \frac{\Delta(s t)}{\delta(s t)} d \mu(t) d \mu(s) d \dot{\lambda}(\dot{x}) \\
& =\int_{G / H} \frac{1}{\rho(x)} \int_{H} \kappa(x s) d \mu(s) \int_{H}|f(x t)| \frac{\Delta(t)}{\delta(t)} d \mu(t) d \dot{\lambda}(\dot{x}) \\
& \leq \mu(W) \int_{G / H} \frac{1}{\rho(x)} \int_{H}|f(x t)| \frac{\Delta(t)}{\delta(t)} d \mu(t) d \dot{\lambda}(\dot{x}) \\
& =\mu(W)\|f\| .
\end{aligned}
$$

Here we have used that

$$
\int_{H} \kappa(x s) d \mu(s)=\left\{\begin{array}{lll}
0 & \text { if } & \dot{x} \notin \mathcal{C} \\
\mu(W) & \text { if } & \dot{x} \in \mathcal{C} .
\end{array}\right.
$$


It follows that $T_{\kappa}$ is bounded with $\left\|T_{\kappa}\right\|=\mu(W)$, since we have ' $=$ ' in the above estimates when $f \geq 0$ and $\operatorname{supp} f \subseteq C H$.

We next prove that $T_{\kappa}$ is a module homomorphism. Let $s, t \in H$ then

$$
\begin{aligned}
\left(T_{\kappa}(f . s)\right)(t) & =\left\langle f . s . t^{-1}, \kappa\right\rangle \delta\left(t^{-1}\right) \\
& =\left\langle f .\left(t s^{-1}\right)^{-1}, \kappa\right\rangle \frac{\delta\left(s t^{-1}\right)}{\delta\left(s t^{-1}\right)} \delta\left(t^{-1}\right) \\
& =T_{\kappa} f\left(t s^{-1}\right) \delta\left(s^{-1}\right) \\
& =\left(\left(T_{\kappa} f\right) . s\right)(t) .
\end{aligned}
$$

Now for $f=\kappa$ we get

$$
\begin{aligned}
\int_{H} T_{\kappa}(\kappa)(t) d \mu(t) & =\int_{G / H} \int_{H} \kappa(x s) d \mu(s) \frac{1}{\rho(x)} \int_{H} \kappa(x t) \frac{\Delta(t)}{\delta(t)} d \mu(t) d \dot{\lambda}(\dot{x}) \\
& =\mu(W)\|\kappa\| .
\end{aligned}
$$

To show that $\operatorname{supp}\left(T_{\kappa}(\kappa)\right) \subseteq V$, suppose that $\kappa(x t) \kappa(x) \neq 0$, and let $\{c\}=$ $C \cap(x H)$. Then $x t=c s^{\prime} \quad x=c s^{\prime \prime}$ for (unique) $s^{\prime}, s^{\prime \prime} \in W$, so $t \in W^{-1} W \subseteq$ $V$. It follows that $T_{\kappa}(\kappa)(t)=\int_{G} \kappa(x t) \kappa(x) d \lambda(x) \Delta(t) \delta\left(t^{-1}\right)$ vanishes outside $V$. If we put

$$
T=\frac{1}{\mu(W)} T_{\kappa} \quad \text { and } \quad f=\frac{1}{\|\kappa\|} \kappa
$$

we have (a), .., (d) fulfilled.

It is now an easy matter to prove:

Theorem III.3. Let $G$ be a locally compact group and let $H$ be a closed subgroup. Then $L_{1}(G)_{H}$ is a generator of ess-mod- $H$.

Proof. First we note that the functions $T f$ in the statement of Lemma III.2 form an approximate identity for $L_{1}(H)$, inductively ordered by neighborhoods of $e$ in $H$ and bounded by 1. A standard argument now finishes the proof. Let $h \in L_{1}(H)$ with $\|h\|=1$ and let $\varepsilon>0$. Choose $T \in \mathbf{h}_{H}\left(L_{1}(G), L_{1}(H)\right)$ and $f \in L_{1}(G)$ with $\|T\|=\|f\|=1$ such that $\|T f * h-h\| \leq \varepsilon$. Since $T \in \mathbf{h}_{H}\left(L_{1}(G), L_{1}(H)\right)$ we have $\|T(f * h)-h\| \leq \varepsilon$. Choose now inductively $T_{n}$ and $f_{n}$, so that $\left\|T_{n}\right\|=\left\|f_{n}\right\|=1$ and

$$
\left\|h-\sum_{i=0}^{n} 2^{-i} T_{i} f_{i}\right\| \leq 2^{-(n+1)} \quad(n=0,1, \ldots),
$$

so that

$$
h=\sum_{i=0}^{\infty} 2^{-i} T_{i} f_{i}
$$


It follows that the canonical map $\mathbf{h}_{H}\left(L_{1}(G), L_{1}(H)\right) \widehat{\otimes} L_{1}(G)$ given by $T \otimes f \rightarrow$ $T f$ is surjective, that is, $L_{1}(G)_{H}$ is a generator.

By the general theory of $[\mathbf{G} 2]$ the module $L_{1}(G)_{H}$ thus qualifies as an equivalence module giving Morita equivalence between $L_{1}(H)$ and the corresponding derived algebra $\mathcal{E}$. As a Banach space $\mathcal{E}=P \underset{H}{\widehat{\otimes}} P^{\sharp}$ with $P^{\sharp}=$ $\operatorname{clspan}\left\{a . \varphi \mid \varphi \in \mathbf{h}_{H}\left(L_{1}(G), L_{1}(H)\right), a \in L_{1}(H)\right\}$ and $P=L_{1}(G)$ and the product

$$
(f \underset{H}{\otimes} a \varphi)\left(f^{\prime} \underset{H}{\otimes} a^{\prime} \varphi^{\prime}\right)=f a \varphi\left(f^{\prime}\right) \underset{H}{\otimes} a^{\prime} \varphi^{\prime}
$$

Since $L_{1}(G)_{H}$ is flat, the algebra $\mathcal{E}$ is $\mathrm{H}$-unital as a Banach algebra and short exact sequences in ind $\mathcal{E}-\mathbf{m o d}$ will be short exact in Ban, see [G2]. Using the descriptions of $L_{1}(G) \underset{H}{\widehat{\otimes}}-$ and $\mathbf{h}_{H}\left(L_{1}(G),-\right)$ in [R1, Sections 9 \& 10] it is possible to express $\mathcal{E}$ as vector valued function space of some (involved) cross product type. However, we shall not go into this, since in the next section we shall find a simpler description of induced modules. At this stage we just note:

Corollary III.4 (Imprimitivity Theorem, preversion). Let $X \in G$-mod and let $\mathcal{E}$ be the Banach algebra derived from $L_{1}(G)_{H}$. Then $X$ is strictly induced from $H-\bmod$ via $L_{1}(G)_{H}$, if and only if $X \in \operatorname{ind} \mathcal{E}-\bmod$ with the two module actions being compatible, i.e.

$$
f((g \underset{H}{\otimes} a \varphi) \cdot x)=((f g) \underset{H}{\otimes} a \varphi) \cdot x
$$

for all $f, g \in L_{1}(G), a \in L_{1}(H), \varphi \in \mathbf{h}_{H}\left(L_{1}(G), L_{1}(H)\right)$.

Example III.5. Let $G$ be discrete and let $H=\{e\}$. Then $\mathcal{E}=\mathcal{N}\left(\ell_{1}(G)\right)$, the nuclear operators on the Banach space $\ell_{1}(G)$. The strictly induced $\ell_{1}(G)$ modules are precisely the modules of the form $\ell_{1}(G) \widehat{\otimes} E$, where $E$ is some Banach space, that is, the (relatively) free $\ell_{1}(G)$-modules (see $[\mathbf{H e l}]$ ). Hence an $\ell_{1}(G)$-module $X$ is (relatively) free if and only if $X$ is in ind- $\mathcal{N}\left(\ell_{1}(G)\right)$ $\bmod$ in such a way that

$$
f((g \otimes m) \cdot x)=(f g \otimes m) \cdot x
$$

for all $f, g \in \ell_{1}(G), m \in \ell_{\infty}(G), x \in X$.

\section{Systems of imprimitivity.}

In this section we want to show how the conclusion of Corollary III.4 can be implemented as the existence of 'systems of imprimitivity'. Throughout we 
are dealing with a fixed locally compact group $G$ and a fixed closed subgroup $H$. The Banach algebra derived from $L_{1}(G)_{H}$ will be denoted $\mathcal{E}$ and referred to as the imprimitivity algebra. We shall follow Rieffel in comparing $\mathcal{E}$ to a certain crossed product group algebra. Let $Y \in H-\bmod$ and consider the strictly induced $X=L_{1}(G) \widehat{\otimes} Y$. The $X$ is naturally a Banach module over the endomorphism algebra $\mathbf{h}_{H}\left(L_{1}(G), L_{1}(G)\right)$ simply by

$$
T .(f \underset{H}{\otimes} y)=(T f) \underset{H}{\otimes} y \quad\left(T \in \mathbf{h}_{H}\left(L_{1}(G), L_{1}(G)\right), f \in L_{1}(G), y \in Y\right) .
$$

With respect to the b.s.o. topology on $\mathbf{h}_{H}\left(L_{1}(G), L_{1}(G)\right)$ this action is continuous. The strategy is now to show that the canonical image of $\mathcal{E}$ in $\mathbf{h}_{H}\left(L_{1}(G), L_{1}(G)\right)$ is contained in the b.s.o. closure of some tangible subalgebra of $\mathbf{h}_{H}\left(L_{1}(G), L_{1}(G)\right)$. This approach is feasible precisely because representations of $\mathcal{E}$ which are induced from representations of $L_{1}(H)$ factor through $\mathbf{h}_{H}\left(L_{1}(G), L_{1}(G)\right)$ :

$$
(g \underset{H}{\otimes} a \varphi) \cdot(f \underset{H}{\otimes} y)=g \underset{H}{\otimes} a \varphi(f) y
$$

for all $f, g \in L_{1}(G), a \in L_{1}(H), \varphi \in \mathbf{h}_{H}\left(L_{1}(G), L_{1}(H)\right), y \in Y$.

The Banach algebras $L_{1}(G)$ and $C_{0}(G / H)$ (the commutative Banach algebra of continuous functions on the locally compact space $G / H$ vanishing at $\infty$ ) have isometric copies in $\mathbf{h}_{H}\left(L_{1}(G), L_{1}(G)\right)$ as convolution operators and multiplication operators respectively. We shall not distinguish notationally between these Banach algebras and their isometric copies. We use the notation $T \cdot S$ for the operator product in $\mathbf{h}_{H}\left(L_{1}(G), L_{1}(G)\right)$.

Definition IV.1. The crossed product $L_{1}(G) \times C_{0}(G / H)$ is the subalgebra of $\mathbf{h}_{H}\left(L_{1}(G), L_{1}(G)\right)$ generated by $L_{1}(G) \cdot C_{0}(G / H)$.

The fundamental observation of Rieffel is that systems of imprimitivity can be described in terms of a module action of $L_{1}(G) \times C_{0}(G / H)$. In carrying through the project of Rieffel in a non-involutive setting the basic technical step is the proposition below. We denote the canonical image of $\mathcal{E}$ in $\mathbf{h}_{H}\left(L_{1}(G), L_{1}(G)\right)$ by $\widetilde{\mathcal{E}}$. It is given by

$$
(F \underset{H}{\otimes} S) f=F * S f \quad\left(F, f \in L_{1}(G), S \in \mathbf{h}_{H}\left(L_{1}(G), L_{1}(H)\right)\right)
$$

where $*$ is convolution of measures viewing as before $L_{1}(H)$ as Borel measures on $G$.

Proposition IV.2. $\widetilde{\mathcal{E}}$ is contained in the b.s.o. closure of $L_{1}(G) \times C_{0}(G / H)$.

The proof of the proposition consists of several steps. We start by noting the following elementary fact. The symbol $\dot{\mathcal{U}}(e)$ denotes the filter of neighborhoods of the identity $e$. 
Lemma IV.3. Let $K \subseteq G$ be compact. Then

$$
K=\bigcap_{V \in \mathcal{U}(e)} K V .
$$

If $O \supseteq K$ is open, then there is $V \in \dot{\mathcal{U}}(e)$ such that $K V \subseteq O$.

Proof. Let $x \in \bigcap K V$, say $x=k_{v} v$. By compactness we may assume that $k_{v} \rightarrow k \in K$ along $\dot{\mathcal{U}}(e)$. But then $x=k \in K$. Suppose we cannot find $V$. Then $\{K V \cap(G \backslash O) \mid V \in \dot{\mathcal{U}}(e)\}$ has the finite intersection property and thus has non-empty intersection, since $G$ is locally compact. But this contradicts the first part of the statement.

In dealing with operators from $\mathbf{h}_{H}\left(L_{1}(G), L_{1}(H)\right)$ the next lemma shows that it suffices to look at a special kind:

Lemma IV.4. Let $S \in \mathbf{h}_{H}\left(L_{1}(G), L_{1}(H)\right)$. Then $S$ is a s.o. limit of a bounded net of operators in $\mathbf{h}_{H}\left(L_{1}(G), L_{1}(H)\right)$ of the form

$$
\left(S_{\phi} f\right)(t)=\left\langle f . t^{-1}, \phi\right\rangle \delta\left(t^{-1}\right) \quad\left(f \in L_{1}(G), t \in H\right)
$$

where $\phi: G \rightarrow \mathbb{C}$ is a bounded continuous function. Furthermore

$$
\left\|S_{\phi}\right\|=\sup _{u \in G} \int_{H}|\phi(u t)| d \mu(t) .
$$

Proof. Let $a \in L_{1}(G)$ and consider the operator $f \rightarrow S(a * f)$. Defining a continuous vector valued function $\Phi: G \rightarrow L_{1}(H)$ by $\Phi(u)=S(a . u)$ we have

$$
S(a * f)=\int_{G} f(u) \Phi(u) d \lambda(u)
$$

where the integral converges as a Bochner integral. Let $b \in C_{c}(H)$. Then $b * L_{1}(H) \subseteq C_{u}(H)$ and

$$
b * S(a * f)=\int_{G} f(u) b * \Phi(u) d \lambda(u) .
$$

Using that $S$ is a module homomorphism we see that $\Phi(u t)=\Phi(u) . t \quad(u \in$ $G, t \in H)$. Let $e \in G$ be the neutral element and put

$$
\phi(u)=b * \Phi(u)(e) \quad(u \in G) .
$$


This defines a continuous function bounded by $\|S\|\|a\| \sup _{t \in H}\{\delta(t)|b(t)| \mid t \in$ $H\}$. Note that the definition makes sense, since $b * \Phi(u) \in C_{u}(H)$ for each $u \in G$. Now for each $t \in H$

$$
\begin{aligned}
b * S(a * f)(t) & =[b * S(a * f)] \cdot t^{-1}(e) \delta\left(t^{-1}\right) \\
& =b * S\left(a * f \cdot t^{-1}\right)(e) \delta\left(t^{-1}\right) \\
& =\int_{G}\left(f \cdot t^{-1}\right)(u)(b * \Phi(u))(e) d \lambda(u) \delta\left(t^{-1}\right) \\
& =\left\langle f . t^{-1}, \phi\right\rangle \delta\left(t^{-1}\right) .
\end{aligned}
$$

If we let $b$ 's consist of a bounded approximate identity for $L_{1}(H)$ and $a$ 's consist of a bounded approximate identity for $L_{1}(G)$ we get a net $S_{i} \in$ $\mathbf{h}_{H}\left(L_{1}(G), L_{1}(H)\right)$ with the desired properties. To estimate the operator norm we first note that

$$
\|b * S(a \cdot)\| \geq \limsup _{\gamma}\left\|b * S\left(a * e_{\gamma} \cdot u\right)\right\|=\|b * \Phi(u)\|,
$$

for each $u \in G$, where $\left(e_{\gamma}\right)_{\Gamma}$ is a standard bounded approximate identity for $L_{1}(G)$. It follows that

$$
\begin{aligned}
\left\|S_{\phi}\right\| & =\sup _{u \in G}\|b * \Phi(u)\| \\
& =\sup _{u} \int_{H}|b * \Phi(u)(t)| d \mu(t) \\
& =\sup _{u} \int_{H}|\phi(u t)| d \mu(t) .
\end{aligned}
$$

The next lemma shows that the operators of the previous lemma may be defined in terms of functions with compact support.

Lemma IV.5. Let $S: L_{1}(G) \rightarrow L_{1}(H)$ have the form

$$
(S f)(t)=\left\langle f . t^{-1}, \phi\right\rangle \delta\left(t^{-1}\right) \quad(t \in H),
$$

for some $\phi \in C_{b}(G)$ with $\sup _{u \in G} \int_{H}|\phi(u t)| d t=\|S\|<\infty$. Then $S$ is a b.s.o. limit of operators of the form

$$
\left(S_{\psi} f\right)(t)=\left\langle f . t^{-1}, \psi\right\rangle \delta\left(t^{-1}\right) \quad(t \in H),
$$

where $\psi \in C_{c}(G)$.

Proof. Fix a finite set $\mathfrak{F} \subseteq L_{1}(G)$ and $\varepsilon>0$ and choose a compact set $K$ such that

$$
\int_{G \backslash K}|\phi(u)| \int_{H}|f(u t)| \frac{\Delta(t)}{\delta(t)} d \mu(t) d \lambda(u)<\varepsilon
$$


for all $f \in \mathfrak{F}$. Let $\Gamma$ be the net of compactly supported continuous functions $\chi: G \rightarrow[0,1]$ with the usual ordering and choose $\chi_{0} \in \Gamma$ with $\chi_{0}(K) \subseteq\{1\}$. Then for any $\chi \geq \chi_{0}$ we have, putting $\psi_{\chi}=\chi \phi$ (pointwise multiplication), that for all $f \in \mathfrak{F}$

$$
\begin{aligned}
\left\|\left(S-S_{\psi_{\chi}}\right)(f)\right\| & =\int_{H}\left|\left\langle f \cdot t^{-1}, \phi-\psi_{\chi}\right\rangle\right| \delta\left(t^{-1}\right) d \mu(t) \\
& \leq \int_{G}|(\phi-\chi \phi) u| \int_{H}|f(u t)| \frac{\Delta(t)}{\delta(t)} d \mu(t) d \lambda(u) \\
& \leq \int_{G \backslash K}|\phi(u)| \int_{H}|f(u t)| \frac{\Delta(t)}{\delta(t)} d \mu(t) d \lambda(u) \\
& <\varepsilon
\end{aligned}
$$

Let $\phi \in C_{c}(G)$ and recall that the right module action of $G$ on $\phi$, viewed as an element $\phi \in L_{1}(G)$, is given by

$$
\phi . x=\phi\left(\cdot x^{-1}\right) \Delta\left(x^{-1}\right) \quad(x \in G) .
$$

We want to show that for fixed $f \in L_{1}(G)$ the map $x \rightarrow S_{\phi . x}(f): G \rightarrow L_{1}(H)$ is continuous. First we prove.

Lemma IV.6. Let $\phi \in C_{c}(G)$ and define a function $\Phi: G \rightarrow \mathbb{C}$ by

$$
\Phi(u)=\int_{H} \phi(u t) d \mu(t) \quad(u \in G) .
$$

Then $\Phi$ is bounded and left uniformly continuous.

Proof. Let $\operatorname{supp} \phi=K$. By left invariance of Haar measure on $H$ we have $\Phi(u)=\Phi(u s) \quad(u \in G, s \in H)$ and $\Phi$ is supported on $K H$. Define $\widetilde{\Phi}$ : $G / H \rightarrow \mathbb{C}$ by $\widetilde{\Phi}(\dot{u})=\Phi(u), \dot{u}=u H$. Then $\widetilde{\Phi}$ is continuous if and only if $\Phi$ is continuous. Fix $v \in G$. Since $\phi$ is compactly supported, it is uniformly continuous, so given $\varepsilon>0$ there is a neighborhood $W=W^{-1}$ so that if $u \in v W$ then

$$
\|\phi(u \cdot)-\phi(v \cdot)\|_{\infty} \leq \frac{\varepsilon}{\mu\left(v^{-1} K\right)+1} .
$$

Hence

$$
\begin{aligned}
|\Phi(u)-\Phi(v)| & \leq \int_{H}|\phi(u t)-\phi(v t)| d \mu(t) \\
& \leq \frac{\varepsilon \mu\left(W v^{-1} K\right)}{\mu\left(v^{-1} K\right)+1}
\end{aligned}
$$


since $\operatorname{supp}(\phi(u \cdot)-\phi(v \cdot)) \subseteq W v^{-1} K$. By outer regularity of $\mu$ and Lemma IV.3 we may ensure that $W$ is chosen so that $\mu\left(W v^{-1} K\right) \leq \mu\left(v^{-1} K\right)+1$. Hence, if $u \in v W$, then $|\Phi(u)-\Phi(v)| \leq \varepsilon$. Since $\widetilde{\Phi}$ is continuous and supported on $\dot{K}$, it is bounded, so that $\Phi$ itself is bounded. A standard compactness argument then shows that $\Phi$ is uniformly continuous from the left.

In the following lemma we identify functions on $G$ which are constant on cosets $u H$ with functions on $G / H$.

Lemma IV.7. Let $\phi \in C_{c}(G)$. The map

$$
\Psi: x \rightarrow\left(u \rightarrow \int_{H} \phi(u t x) d \mu(t)\right): G \rightarrow C_{c}(G / H)
$$

is continuous.

Proof. Put $\operatorname{supp} \phi=K$, let $\varepsilon>0$ and let $x \in G$. Let $V$ be a compact neighborhood of $e$ in $G$ and choose $W \subseteq V$ so that, if $y \in W x$, then

$$
\|\phi(\cdot x)-\phi(\cdot y)\|_{\infty} \leq \frac{\varepsilon}{\mu\left(K x^{-1} V^{-1}\right)+1} .
$$

If $y \in W x$, then $\operatorname{supp} \phi(\cdot y) \bigcup \operatorname{supp} \phi(\cdot x) \subseteq K x^{-1} W^{-1} \subseteq K x^{-1} V^{-1}$. It follows that

$$
\begin{aligned}
\|\Psi(x)-\Psi(y)\|_{\infty} & \leq \sup _{u} \int_{H}|\phi(u t x)-\phi(u t y)| d t \\
& \leq \frac{\varepsilon}{\mu\left(K x^{-1} V^{-1}\right)+1}\left(\mu\left(K x^{-1} V^{-1}\right)+1\right) \\
& =\varepsilon .
\end{aligned}
$$

We are now in the position to prove Proposition IV.2.

Proof of Proposition IV.2. First note that $L_{1}(G)$ is contained in the b.s.o. closure of $L_{1}(G) \times C_{0}(G / H)$, since convolution by $F \in L_{1}(G)$ is equal to the b.s.o. limit of a net $\left(F \cdot P_{\gamma}\right)$ where $P_{\gamma}$ is multiplication by a bounded approximate identity in the commutative Banach algebra $C_{0}(G / H)$. Now for $\phi \in C_{c}(G)$ we define two operators $S_{\phi} \in \mathbf{h}_{H}\left(L_{1}(G), L_{1}(H)\right)$ and $T_{\phi} \in$ $\mathbf{h}_{H}\left(L_{1}(G), L_{1}(G)\right)$ by

$$
\begin{aligned}
S_{\phi} f(t) & =\left\langle f \cdot t^{-1}, \phi\right\rangle \delta\left(t^{-1}\right) & & \left(f \in L_{1}(G), t \in H\right) \\
T_{\phi} f(x) & =\left\langle f \cdot x^{-1}, \phi\right\rangle \Delta\left(x^{-1}\right) & & \left(f \in L_{1}(G), x \in G\right) .
\end{aligned}
$$


We have already noted that $\left\|S_{\phi}\right\|=\sup _{u} \int_{H}|\phi(u t)| d t$. Similarly, from

$$
\begin{aligned}
T_{\phi} f(x) & =\int_{G} f(u x) \phi(u) d u \\
& =(\check{\phi} * f)(x)
\end{aligned}
$$

where $\check{\phi}(u)=\phi\left(u^{-1}\right) \Delta u^{-1}$, we see that $\left\|T_{\phi}\right\|=\|\phi\|_{1}$ and that $T_{\phi}$ is in the b.s.o. closure of $L_{1}(G) \times C_{0}(G / H)$. Let now $F, f \in L_{1}(G), P \in C_{0}(G / H)$ and let $T_{\phi}$ and $S_{\phi}$ be as above. The the $L_{1}(G)$-valued function

$$
x \rightarrow \frac{1}{\rho(x)} F . x * S_{\phi . x}(f)
$$

is independent of $x \in \dot{x}=x H$ and we have the following formula

$$
F \cdot P \cdot T_{\phi}(f)=\int_{G / H} P(\dot{x}) \frac{1}{\rho(x)} F \cdot x * S_{\phi . x} f d \dot{\lambda}(\dot{x}) .
$$

To see that (IV.1) is independent of the representative, we just calculate

$$
\begin{aligned}
\frac{1}{\rho(x)} F . x * S_{\phi . x} f & =\frac{1}{\rho(x)} \int_{H} F .(x t) S_{\phi . x} f(t) d \mu(t) \\
& =\frac{1}{\rho(x)} \int_{H} F .(x s t) S_{\phi . x} f(s t) d \mu(t)
\end{aligned}
$$

for any $s \in H$. Now

$$
\begin{aligned}
S_{\phi . x}(f)(s t) & =\left\langle f .(s t)^{-1}, \phi \cdot x\right\rangle \delta(s t)^{-1} \\
& =\left\langle f . t^{-1}, \phi \cdot(x s)\right\rangle \Delta(s) \delta(s t)^{-1} \\
& =\left\langle f . t^{-1}, \phi \cdot(x s)\right\rangle \delta\left(t^{-1}\right) \frac{\Delta(s)}{\delta(s)} \\
& =S_{\phi \cdot(x s)} f(t) \frac{\Delta(s)}{\delta(s)}
\end{aligned}
$$

where we have used

$$
\begin{aligned}
\left\langle f \cdot x^{-1}, \phi\right\rangle & =\int_{G} f(u x) \Delta(x) \phi(u) d \lambda(u) \\
& =\int_{G} f(u) \phi\left(u x^{-1}\right) d \lambda(u) \\
& =\langle f, \phi \cdot x\rangle \Delta(x) .
\end{aligned}
$$


Continuing, we get

$$
\begin{aligned}
\frac{1}{\rho(x)} F . x * S_{\phi \cdot x} f & =\frac{1}{\rho(x)} \int_{H} F \cdot(x s t) S_{\phi \cdot(x s)} f(t) \frac{\Delta(s)}{\delta(s)} d \mu(t) \\
& =\frac{1}{\rho(x)} \frac{\Delta(s)}{\delta(s)} F \cdot(x s) * S_{\phi \cdot(x s)} f \\
& =\frac{1}{\rho(x s)} F \cdot(x s) * S_{\phi \cdot(x s)} f .
\end{aligned}
$$

Now

$$
\begin{aligned}
F * P\left(T_{\phi} f\right) & =\int_{G} F \cdot x P\left(T_{\phi} f\right)(x) d \lambda(x) \\
& =\int_{G / H} P(\dot{x}) \frac{1}{\rho(x)} \int_{H} \frac{\Delta(t)}{\delta(t)}\left\langle f .(x t)^{-1}, \phi\right\rangle \Delta(x t)^{-1} F . x t d \mu(t) d \dot{\lambda}(\dot{x}) \\
& =\int_{G / H} P(\dot{x}) \frac{1}{\rho(x)} \int_{H}(F . x) \cdot t\left\langle f . t^{-1}, \phi \cdot x\right\rangle \delta\left(t^{-1}\right) d \mu(t) d \dot{\lambda}(\dot{x}) \\
& =\int_{G / H} P(\dot{x}) \frac{1}{\rho(x)}(F \cdot x) *\left(S_{\phi . x} f\right) d \dot{\lambda}(\dot{x}),
\end{aligned}
$$

thus proving (IV.2).

If we choose $\phi$ compactly supported, then, by Lemma IV.4 and Lemma IV.5, the function in (IV.1) is continuous. Hence if we choose for each compact neighborhood $K$ of $\dot{e}$ in $G / H$ a function $P_{K} \in C_{0}(G / H)$ with $\operatorname{supp} P_{K} \subseteq K$ and $\int_{G / H} P_{K}(\dot{x}) d \dot{\lambda}(\dot{x})=1$ we see, combining (IV.1) and (IV.2), that the operator

$$
f \rightarrow F * S_{\phi} f
$$

is the b.s.o.-limit of the net in $\mathbf{h}_{H}\left(L_{1}(G), L_{1}(G)\right)$

$$
f \rightarrow \rho(e) F * P_{K}\left(T_{\phi} f\right)
$$

ordered by inclusion of $K$ 's. Combining with Lemma IV.4 and Lemma IV.5 we have that the image of each elementary tensor in $\mathcal{E}$ is in the b.s.o.-closure of $L_{1}(G) \times C_{0}(G / H)$, finishing the proof of Proposition IV.2.

We can now state the Imprimitivity Theorem in terms of systems of imprimitivity analogous to those of Rieffel. First we need to describe the topology in which approximations take place.

Definition IV.8. Let $W \in C_{0}(G / H)-\bmod$, and assume that $W$ is also in $G$-mod. We say that the action of $C_{0}(G / H)$ is $L_{1}(G)$-continuous, if the canonical map

$$
L_{1}(G) \widehat{\otimes} C_{0}(G / H) \rightarrow \mathcal{B}(W)
$$


is continuous with respect to the s.o. topology on $\mathcal{B}(W)$ and the topology on $L_{1}(G) \widehat{\otimes} C_{0}(G / H)$ given by convergence of bounded nets in seminorms: $|F \otimes P|_{f}=\|F * P f\|$, that is the b.s.o. topology of the canonical image $L_{1}(G) \times C_{0}(G / H)$ in $\mathcal{B}\left(L_{1}(G)\right)$.

Theorem IV.9 (Imprimitivity Theorem). Let $X \in G$-mod. Then $X$ is essentially induced from $H-\bmod$ via $L_{1}(G)$ if and only if $X$ is also an essential ( $c f$. Sect. I) $C_{0}(G / H)$-module satisfying the two conditions:

(i) $\quad u .(P . x)=(u . P) .(u . x) \quad\left(u \in G, P \in C_{0}(G / H), x \in X\right)$

(ii) $C_{0}(G / H)$ acts $L_{1}(G)$-continuously on $X$,

where the action of $G$ on $C_{0}(G / H)$ is given by $(u . P)(g)=P\left(u^{-1} g\right)$. If $X$ is essentially induced from $H-\bmod$ via $L_{1}(G)$, then the inducing is automatically faithful.

Proof. Suppose $X$ is essentially induced from $M \in H-\bmod$. By the uniqueness of the action of $\mathbf{h}_{H}\left(L_{1}(G), L_{1}(G)\right)$, (Definition II.1), we see that (i) holds, since it holds when $\mathbf{h}_{H}\left(L_{1}(G), L_{1}(G)\right)$ acts on $L_{1}(G) \underset{H}{\widehat{\otimes}} M$. Similarly, since the action of $\mathbf{h}_{H}\left(L_{1}(G), L_{1}(G)\right)$ on $L_{1}(G) \underset{H}{\widehat{\otimes}} M$ is b.s.o. continuous we have (ii) fulfilled.

Conversely, suppose that (i) and (ii) are satisfied. Since the crossed product has a bounded approximate identity its b.s.o. closure contains the identity operator in $\mathbf{h}_{H}\left(L_{1}(G), L_{1}(G)\right)$. More precisely, a net of operators of the form $F \cdot P \cdot T_{\phi}$ b.s.o. converges to the identity in $\mathbf{h}_{H}\left(L_{1}(G), L_{1}(G)\right)$ when $F$ 's are chosen as a bounded approximate identity in the group algebra $L_{1}(G), P$ 's are chosen to form a bounded approximate identity of compactly supported functions in the Banach algebra $C_{0}(G / H)$ and $\phi$ 's are chosen compactly supported and continuous, so that $\check{\phi}$ 's form a bounded approximate identity in $L_{1}(G)$. By the formula (IV.2), we see that the b.s.o. closure of operators of the form $f \rightarrow F * S_{\phi}(f)$ contains the identity in $\mathbf{h}_{H}\left(L_{1}(G), L_{1}(G)\right)$. But the image of the imprimitivity algebra $\mathcal{E}$ in $\mathbf{h}_{H}\left(L_{1}(G), L_{1}(G)\right)$ is a left ideal, so, taken together, the b.s.o. closure of $L_{1}(G) \times C_{0}(G / H)$ is all of $\mathbf{h}_{H}\left(L_{1}(G), L_{1}(G)\right)$. By (i) we see that the actions of $L_{1}(G)$ and $C_{0}(G / H)$ on $X$ respect the multiplication in $L_{1}(G) \times C_{0}(G / H)$ and by (ii) we can extend this action by b.s.o. continuity to a Banach module action of $\mathbf{h}_{H}\left(L_{1}(G), L_{1}(G)\right)$ on $X$. In particular $X \in \mathcal{E}$-mod. Let $Y=L_{1}(G)^{\sharp} \widehat{\otimes} X$, where $\sharp$ is defined in Sect. I. Then the canonical map $\mu: L_{1}(G) \underset{H}{\widehat{\otimes} Y} \rightarrow X$ has dense range, as for instance can be seen by b.s.o. 
continuity, so in the diagrams

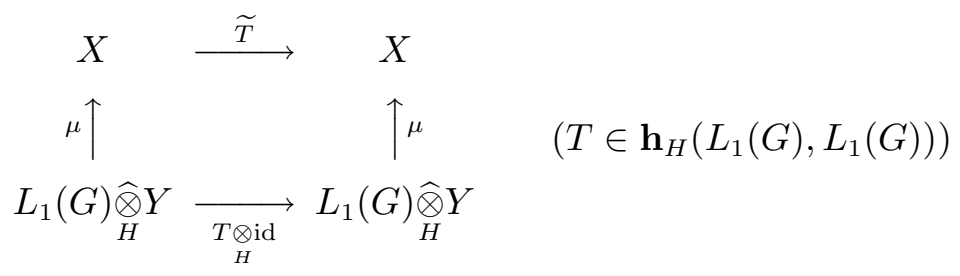

the maps $\widetilde{T}$ are unique. Hence the conditions of the abstract imprimitivity theorem are satisfied.

To prove that $X$ is faithfully induced we must show that the multiplication $\mu: \underset{\mathcal{E}}{\widehat{\otimes}} \underset{\mathcal{E}}{\widehat{a}} X \rightarrow X$ is $1-1$. Hence, suppose that $\sum_{i=1}^{\infty} e_{i} . x_{i}=0$ with $e_{i}=$ $F_{i} \underset{H}{\otimes} S_{i}, F_{i} \in L_{1}(G), S_{i} \in L_{1}(G)^{\sharp}, x_{i} \in X, \Sigma\left\|F_{i}\right\|\left\|S_{i}\right\|\left\|x_{i}\right\|<\infty$. Let $F \cdot P \cdot T_{\phi} \in \mathbf{h}_{H}\left(L_{1}(G), L_{1}(G)\right)$ be one of the operators previously considered with $P$ compactly supported. Since $\left\|S_{\phi . x}\right\|=\sup _{u \in G} \int_{H}\left|\phi\left(u t x^{-1}\right)\right| \Delta\left(x^{-1}\right) d \mu(t)$, it follows from Lemma IV.7 that

$$
\sup \left\{\left\|\frac{1}{\rho(x)} F \cdot x * S_{\phi \cdot x}\right\| \mid \dot{x} \in \operatorname{supp} P\right\}=K<\infty .
$$

Let $L=\int_{G / H}|P(\dot{x})| d \dot{\lambda}(\dot{x})$. Then $L<\infty$, since $P$ is compactly supported. Let $\varepsilon>0$ and choose $N$ so that

$$
\left\|\sum_{i=1}^{N} e_{i} \cdot x_{i}\right\| \leq \frac{\varepsilon}{2 K L}
$$

and

$$
\sum_{i=N+1}^{\infty}\left\|e_{i}\right\|\left\|x_{i}\right\| \leq \frac{\varepsilon}{2\left\|F \cdot P \cdot T_{\phi}\right\|} .
$$

Then

$$
\begin{aligned}
& \left\|F \cdot P \cdot T_{\phi} \cdot \sum e_{i} \underset{\mathcal{E}}{\otimes} x_{i}\right\| \\
& \leq\left\|\sum_{1}^{N} \int_{G / H} P(\dot{x}) \frac{1}{\rho(x)}(F . x) * S_{\phi . x}\left(F_{i}\right) d \dot{\lambda}(\dot{x}) \underset{H}{\underset{\mathcal{E}}{S_{i}} \underset{\mathcal{E}}{\otimes} x_{i} \|}\right\|+\frac{\varepsilon}{2} .
\end{aligned}
$$

The integral $\int_{G / H} P(\dot{x}) \frac{1}{\rho(x)} F \cdot x * S_{\phi . x}\left(F_{i}\right) d \dot{\lambda} \dot{x}$ is the limit of a net of convex combinations of the form $\sum_{k} \lambda_{k} F \cdot x_{k} * S_{\phi \cdot x_{k}}\left(F_{i}\right)$ where $\left\|F \cdot x_{k} \cdot S_{\phi . x_{k}}\right\| \leq K L$. By the definition of the multiplication in $\mathcal{E}$ we have $F \cdot x_{k} * S_{\phi \cdot x_{k}}\left(F_{i}\right) \underset{H}{\otimes} S_{i} \underset{\mathcal{E}}{\otimes} x_{i}=$ 


$$
\begin{aligned}
\left(F \cdot x_{k} \underset{H}{\otimes} S_{\phi \cdot x_{k}}\right) \underset{\mathcal{E}}{\otimes}\left(F_{i} \underset{H}{\otimes} S_{i}\right) \cdot x_{i}, \text { so } \\
\left\|\sum_{1}^{N}\left(F \cdot P \cdot T_{\phi}\right) \cdot F_{i} \underset{H}{\otimes} S_{i} \underset{\mathcal{E}}{\otimes} x_{i}\right\| \leq K L\left\|\sum_{1}^{N} e_{i} \cdot x_{i}\right\| \leq \frac{\varepsilon}{2} .
\end{aligned}
$$

Altogether, since $\varepsilon>0$ was arbitrary, $\left(F \cdot P \cdot T_{\phi}\right) \cdot \sum_{1}^{\infty} e_{i}{\underset{\mathcal{E}}{\otimes}}_{i} x_{i}=0$. Since operators of the form $F \cdot P \cdot T_{\phi}$ contains a bounded net converging s.o. to the identity in $\mathbf{h}_{H}\left(L_{1}(G), L_{1}(G)\right)$ it follows that $\sum_{1}^{\infty} e_{i} \underset{\mathcal{E}}{\otimes} x_{i}=0$.

Since inducing of modules via $L_{1}(G)$ is automatically faithful, induced modules occur as various completions of an algebraic tensor product. It seems worthwhile to describe the important case of strictly induced modules geometrically. In order to find such a condition we first need a lemma describing the situation for the module $L_{1}(G) \underset{H}{\widehat{\otimes}} Y$.

Lemma IV.10. Let $\mathfrak{F} \subseteq L_{1}(G)$ be a finite set and let $\varepsilon>0$. Then there are sequences $\left(g_{n}\right)$ in $L_{1}(G),\left(S_{n}\right)$ in $\mathbf{h}_{H}\left(L_{1}(G), L_{1}(H)\right)$, and $\left(P_{n}\right)$ in $C_{0}(G / H)$ so that

(1) $\left\|f-\sum g_{n} * S_{n}\left(P_{n} f\right)\right\|<\varepsilon \quad(f \in \mathfrak{F})$.

(2) $\operatorname{supp}\left(P_{m}\right) \cap \operatorname{supp}\left(P_{n}\right)=\varnothing$, when $m \neq n$.

(3) $\left\|g_{n}\right\| \leq 1$ and $\left\|S_{n}\right\| \leq 1 \quad(n \in \mathbb{N})$.

Proof. First we choose $g \in L_{1}(G)$ with $\|g\|=1$ so that $\|f-g * f\|<\frac{\varepsilon}{3}(f \in \mathfrak{F})$. Next, since $\cup_{f \in \mathfrak{F}} \operatorname{supp}(f)$ is $\sigma$-compact and $\dot{\lambda}$ is completion regular, we may choose a sequence of mutually disjoint open sets $\mathcal{O}_{n} \subseteq G / H \quad(n \in \mathbb{N})$ and compact Baire sets $\mathcal{C}_{n} \subseteq \mathcal{O}_{n} \quad(n \in \mathbb{N})$ such that

(IV.3) $\left\|g * f-\sum_{n=1}^{\infty} \int_{\mathcal{C}_{n}} \int_{H} \frac{1}{\rho(x t)} g .(x t) f(x t) d \mu(t) d \dot{\lambda}(\dot{x})\right\|<\frac{\varepsilon}{3} \quad(f \in \mathfrak{F})$.

We look at one of the summands. Since $\mu$ is $H$-translation invariant we have the identity

(IV.4)

$$
\begin{aligned}
& \int_{H} \frac{1}{\rho(x t)} g \cdot(x t) f(x t) d t \\
& =\frac{1}{\mu(W)} \int_{W} \int_{H} \frac{1}{\rho(x s t)} g \cdot(x s t) f(x s t) d \mu(t) d \mu(s) \quad(\dot{\lambda} \text {-almost all } \dot{x})
\end{aligned}
$$

for any $W \subseteq H$ of finite positive measure and all $f \in \mathfrak{F}$. By [Keh] we may choose relatively compact Baire sets $C_{n} \subseteq G$ which are cross sections for $\mathcal{C}_{n}$. 
By refining the countable family $\left(\mathcal{C}_{n}\right)_{n \in \mathbb{N}}$ if necessary, we may choose compact neighborhoods $W_{n}$ of $e$ in $H$ and a sequence $x_{n} \in C_{n}$ so that

$$
\left\|g . x_{n}-g . x s\right\|<\frac{\varepsilon}{3}\|f\| 2^{-n} \quad\left(f \in \mathfrak{F}, x \in C_{n}, s \in W_{n}, n \in \mathbb{N}\right) .
$$

Let $\kappa_{n}$ be the characteristic function of the set $C_{n} W_{n} \subseteq G$ and let $S_{n} \in$ $\mathbf{h}_{H}\left(L_{1}(G), L_{1}(H)\right)$ be the corresponding operator

$$
\left(S_{n} f\right)(t)=\frac{1}{\mu\left(W_{n}\right)}\left\langle f \cdot t^{-1}, \kappa_{n}\right\rangle \delta\left(t^{-1}\right) .
$$

Then, as in the proof of Lemma IV.4, $\left\|S_{n}\right\|=1$ and we have

$$
\left(S_{n} f\right)(t)=\frac{1}{\mu\left(W_{n}\right)} \int_{\mathcal{C}_{n}} \int_{W_{n}} \frac{1}{\rho(x s t)} f(x s t) d \mu(s) d \dot{\lambda}(\dot{x}) \quad(t \in H, f \in \mathfrak{F})
$$

giving

$$
\begin{aligned}
& g \cdot x_{n} * S_{n} f \\
& =\frac{1}{\mu\left(W_{n}\right)} \int_{H} \int_{\mathcal{C}_{n}} \int_{W_{n}} \frac{1}{\rho(x s t)} g \cdot\left(x_{n} t\right) f(x s t) d \mu(s) d \dot{\lambda}(\dot{x}) d \mu(t) .
\end{aligned}
$$

Combining (IV.4) and (IV.5) we see that

$$
\begin{aligned}
& \left\|g \cdot x_{n} * S_{n} f-\int_{\mathcal{C}_{n}} \int_{H} \frac{1}{\rho(x t)} g \cdot x t f(x t) d \mu(t) d \dot{\lambda}(\dot{x})\right\| \\
& <\frac{\varepsilon}{3} 2^{-n} \quad(f \in \mathfrak{F}, n \in \mathbb{N})
\end{aligned}
$$

so that all together

$$
\left\|f-\sum_{n=1}^{\infty} g \cdot x_{n} * S_{n} f\right\|<\varepsilon \quad(f \in \mathfrak{F}) .
$$

We reach the conclusion by noting that, if $P_{n} \in C_{0}(G / H)$ is supported inside $\mathcal{O}_{n}$ with $P_{n}\left(\mathcal{C}_{n}\right)=\{1\}$, then $S_{n} f=S_{n} P_{n} f$ for any $f \in L_{1}(G)$.

We can now describe strictly induced modules.

Theorem IV.11. Let $X \in G$ - mod and assume that $X$ is essentially induced from $Y \in H-\bmod$ via $L_{1}(G)$. Then $X$ is strictly induced from $Y$ if and only if the action of $C_{0}(G / H)$ on $X$ satisfies: There is a constant $C>0$ so that

$$
\sum_{i=1}^{n}\left\|P_{i} x\right\| \leq C\left\|\sum_{i=1}^{n} P_{i} x\right\| \quad(x \in X),
$$


whenever $P_{1}, \ldots, P_{n} \in C_{0}(G / H)$ are mutually disjointly supported.

Proof. From the description of $L_{1}(G) \underset{G}{\widehat{\otimes}}-$ in $[\mathbf{R} 1$, Theorem 10.4] it is obvious that the condition is necessary. To prove sufficiency, let as before $\mathcal{E}$ be the imprimitivity algebra. We want to show that the multiplication $m: \mathcal{E} \widehat{\mathcal{Q}}_{\mathcal{E}} X \rightarrow$ $X$ is bounded below. Let $i: L_{1}(G) \underset{H}{\widehat{\otimes}} Y \rightarrow X$ be the map of the definition

of essential inducing, let $\sum_{k=1}^{K} f_{k} \underset{H}{\otimes} y_{k} \in L_{1}(G) \underset{H}{\otimes} Y$ be a finite tensor and let $\varepsilon>0$. In accordance with Lemma IV.10, choose sequences $g_{n} \in L_{1}(G)$, $S_{n} \in \mathbf{h}_{H}\left(L_{1}(G), L_{1}(H)\right), P_{n} \in C_{0}(G / H)$ such that

$$
\left\|\sum f_{k} \underset{H}{\otimes} y_{k}-\sum_{n, k} g_{n} * S_{n}\left(P_{n} f_{k}\right) \underset{H}{\otimes} y_{k}\right\| \leq \frac{\varepsilon}{\|i\|} .
$$

Put $x=i\left(\sum f_{k} \underset{H}{\otimes} y_{k}\right)$. Then

$$
\begin{aligned}
& \left\|x-m\left(\sum g_{n} \underset{H}{\otimes} S_{n} \underset{\mathcal{E}}{\otimes} P_{n} x\right)\right\| \\
& =\left\|i\left(\sum f_{k} \underset{H}{\otimes} y_{k}-\sum_{n, k} g_{n} * S_{n}\left(P_{n} f_{k}\right) \underset{H}{\otimes} y_{k}\right)\right\| \leq \varepsilon .
\end{aligned}
$$

Since the usual bounded approximate identity for $C_{0}(G / H)$ acts as a bounded approximate identity for $X$, the action being $L_{1}(G)$-continuous, it is clear from the proof of Lemma IV.10 that we may arrange that $\sum P_{n} x$ is arbitrary close to $x$. Since

$$
\begin{aligned}
\sum\left\|g_{n}\right\|\left\|S_{n}\right\|\left\|P_{n} x\right\| & \leq \sum\left\|P_{n} x\right\| \\
& \leq C\left\|\sum P_{n} x\right\|
\end{aligned}
$$

and since elements of the form $i\left(\sum f_{k} \underset{H}{\otimes} y_{k}\right)$ form a dense subset of $X$ a standard argument shows that $m$ is bounded below.

\section{An illustration.}

As an illustration we consider representations of $\mathbb{R}$. Firstly let us remark that a classification of all bounded strongly continuous representations of $\mathbb{R}$ is a formidable task, since it by the Hille-Yosida theorem amounts to a classification of all densely defined closed operators on arbitrary Banach spaces satisfying the Hille-Yosida resolvent estimate. 
We will apply the imprimitivity theorem to the situation $G=\mathbb{R}$ and $H=\mathbb{Z}$ so that $G / H \cong \mathbb{T}$, the circle group. A representation of $\mathbb{Z}$ corresponds to a doubly power bounded operator $T: Y \rightarrow Y$ on a Banach space $Y$. If we form the strictly induced representation we get $L_{1}(\mathbb{R}) \underset{\mathbb{Z}}{\otimes} Y$ which can be identified with (see [Rie, Theorem 10.4]) the Banach space of functions $f: \mathbb{R} \rightarrow Y$ satisfying $T^{n}(f(s))=f(s-n)$ a.e. for all $n \in \mathbb{Z}$ and normed by $\|f\|=\int_{0}^{1}\|f(s)\| d s$. The action of $\mathbb{R}$ is just that of translation $t . f(s)=$ $f(s-t)$ a.e. $(t \in \mathbb{R})$ so the action of $\mathbb{R}$ is generated by the operator $\frac{d}{d t}$ on $L_{1}(\mathbb{R}) \underset{\mathbb{Z}}{\widehat{\otimes}} Y$.

Now suppose that $X$ is an $\mathbb{R}$-module essentially induced via $L_{1}(\mathbb{R})_{\mathbb{Z}}$. Denote the action of $\mathbb{R}$ by $T(t): X \rightarrow X(t \in \mathbb{R})$. For $P \in C(\mathbb{R} / \mathbb{Z})$ we then have the imprimitivity condition

$$
T(t)(P . x)=(t P) \cdot T(t) x \quad(t \in \mathbb{R})
$$

where $t \rightarrow t P$ is the action of $\mathbb{R}$ by rotation. Let $A$ be the infinitesimal generator of the group $(T(t))_{t \in \mathbb{R}}$, let $x \in \mathcal{D}(A)$ and let $P \in \mathcal{D}\left(\frac{d}{d t}\right)$. (Here $\mathcal{D}(-)$ denotes the domain of an operator.) Since the action of $C(\mathbb{R} / \mathbb{Z})$ is b.s.o. continuous, the left hand side of (V.1) is differentiable at $t=0$. Hence P. $x \in \mathcal{D}(A)$ and we get $\left(\frac{d}{d t} P\right) . x+P . A x=A(P . x)$, which can be interpreted as a Leibnitz-type rule for the operator $A$. In fact, this rule is the imprimitivity condition expressed in terms of the infinitesimal generator.

Theorem V.1. Let $t \rightarrow T(t): \mathbb{R} \rightarrow B(X)$ be a bounded strongly continuous representation of $\mathbb{R}$ with infinitesimal generator $A$, and suppose that $C(\mathbb{R} / \mathbb{Z})$ acts $L_{1}(\mathbb{R})$-continuously on $X$. Then $X$ is essentially induced from $\mathbb{Z}-\bmod$ via $L_{1}(\mathbb{R})_{\mathbb{Z}}$ if and only if for all $x \in \mathcal{D}(A)$ and all $P \in \mathcal{D}\left(\frac{d}{d t}\right)$ we have $P . x \in \mathcal{D}(A)$ and

$$
A(P . x)=\left(\frac{d}{d t} P\right) \cdot x+P . A x .
$$

Proof. To prove the sufficiency, we must verify condition (V.1). This is just a modification of the proof that closed derivations generate groups of automorphisms. Applying (V.2) to $t P$ instead of $P$ we get

$$
A(t P . x)=\left(t \frac{d}{d t} P\right) \cdot x+(t P) \cdot A x
$$


since differentiation commutes with translation. Now

$$
\begin{aligned}
& \frac{d}{d s} T(t-s)((s \cdot P) \cdot T(s) x) \\
& =-T(t-s) A((s P) \cdot T(s) x)+T(t-s)\left(s \frac{d}{d s} P \cdot T(s) x\right) \\
& \quad+T(t-s)((s P) \cdot(A T(s) x))=0,
\end{aligned}
$$

where differentiability follows from b.s.o. continuity of the action of $C(\mathbb{R} / \mathbb{Z})$. On inserting $s=0$ and $s=t$ we get

$$
T(t)(P . x)=(t P) . T(t) x \quad(t \in \mathbb{R}),
$$

which holds globally by density of $\mathcal{D}(A)$ and $\mathcal{D}\left(\frac{d}{d t}\right)$.

Remark. The rule (V.2) can be rewritten as a lifting of derivations. Denote the representation of $C(\mathbb{R} / \mathbb{Z})$ by $\Xi$ and let $\delta_{A}$ be the inner derivation $S \rightarrow A S-S A$ on $B(X)$. Then we have a commutative diagram:

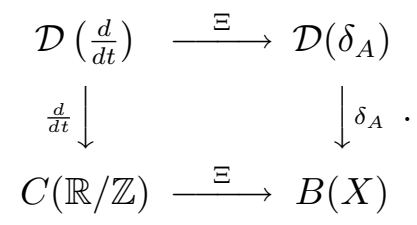

We now focus attention on so-called monomial representations, that is, representations which are induced from 1-dimensional representations of subgroups, see [C\&R, p. 262]. A 1-dimensional bounded representation of $\mathbb{Z}$ corresponds to a complex number of modulus 1 , say $e^{i \alpha}$ for some $\alpha \in \mathbb{R}$. The corresponding strictly induced module is $\{f: \mathbb{R} \rightarrow \mathbb{C} \mid f(t-n)=$ $e^{i n \alpha} f(t)$ (a. e.), $\left.n \in \mathbb{Z},\|f\|=\int_{0}^{1}|f(t)| d t<\infty\right\}$ with translation as action of $\mathbb{R}:(T(t) f)(s)=f(s-t)$ (a. e.). More generally, let $Y$ be a Banach space. From a 1-dimensional representation of $\mathbb{Z}$ given by an augmentation $\ell_{1}(\mathbb{Z}) \rightarrow \mathbb{C}$ we get a representation on $Y$ by identifying $\mathbb{C} \cong \mathbb{C} \operatorname{id}_{Y}$. A representation of $\mathbb{R}$ induced from such a representation of $\mathbb{Z}$ will also be termed monomial.

A fundamental result about doubly power bounded operators $T: Y \rightarrow Y$ is that $\sigma(T)=\{1\} \Rightarrow T=\mathrm{id}_{Y}$, see [Gel]. If $T(t)_{t \in \mathbb{R}}$ is induced from a doubly power bounded operator $T$, then it is easily seen that $\sigma(T(1))=\sigma(T)$. Hence, if an induced representation has $\sigma(T(1))$ a singleton, then it is monomial. Let $A$ be the infinitesimal generator of $T(t)_{t \in \mathbb{R}}$. From the spectral inclusion

$$
\sigma(T(t)) \supseteq \exp (t \sigma(A))
$$


it follows that this can only happen if $\sigma(A)$ is (partly) periodic in the sense that there is $\alpha \in \mathbb{R}$ so that $\sigma(A) \subseteq i(\alpha+2 \pi \mathbb{Z})$.

There are several questions associated with this example. First of all it should be worked out for Lie groups in general. Secondly, the spectral description of the infinitesimal generator is far from satisfactory, as is hinted by the fact that we may have $\sigma(A)=\emptyset$. It would be nice to have a characterization of monomial (induced) representations in terms of spectral properties of the infinitesimal generator. This would probably involve a detailed analysis of spectral subspaces. Thirdly, in the theory of unitary representations monomial representations play an important rôle, since irreducible unitary representations in general can be obtained by inducing irreducible representations from subgroups, see [M4, Sections 3.3.7-8]. What is the importance of monomial representations for general Banach representations?

\section{References}

[Bour] N. Bourbaki, Intégration, Chaps. VII \& VIII, Act. Sci. Ind., 1306, Hermann, Paris, 1963.

[C\&R] C.W. Curtis and I. Reiner, Methods of Representation Theory With Applications to Finite Groups and Orders, Vol. I, Wiley-Interscience, New York, 1981.

[Fell] J.M.G. Fell, Induced representations and Banach* -algebraic bundles, Lecture Notes in Mathematics, 582, Springer-Verlag, Berlin, 1977.

[Fro] G. Frobenius, Über Relationen zwischen den Characteren einer Gruppe und denen ihrer Untergruppen, Sitzber. Preuss. Akac. Wiss., (1898), 501-515.

[Gel] I.M. Gelfand, Zur Theorie der Charaktere der abelscher topologischen Gruppen, Rec. Math. N. S. (Mat. Sbornik), 9 (1941), 49-50.

[Gri] R.I. Grigorchuk, Some results on bounded cohomology, preprint.

[G1] N. Gronbaek, Morita equivalence for Banach algebras, J. Pure Appl. Algebra, 99 (1995), 183-219.

[G2] Morita equivalence for self-induced Banach algebras, Houston J. Math., 22 (1996), 109-140.

[Hel] A.Ya. Helemskiǔ, The homology of Banach and topological algebras, Kluwer, Dordrecht, 1986.

[Hig] D.G. Higman, Induced and produced modules, Canad. J. Math., 7 (1955), 490-508.

[Keh] E.T. Kehlet, Cross sections for quotient maps of locally compact groups, Math. Scand., 55 (1984), 152-160.

[M1] G.W. Mackey, Inprimitivity for representations of locally compact groups I, Proc. Nat. Acad. Sci. U.S.A., 35 (1949), 537-545.

[M2] - Induced representations of locally compact groups, I, Ann. of Math., 55 (1952), 101-139.

[M3] - Induced representations of locally compact groups, II, Ann. of Math., 58 (1953), 193-211. 
[M4] The Theory of Unitary Group Representations, University of Chicago Press, Chicago and London, Chicago Lectures in Mathematics, 1976.

[R1] M.A. Rieffel, Induced Banach representations of Banach algebras and locally compact groups, J. Func. Anal., 1 (1967), 443-491.

[R2] - Induced representations of $C^{*}$-algebras, Adv. Math., 13 (1974), 176-257.

[R3] - Induced representations of rings, Canad. J. Math., 27 (1975), 261-270.

[Rig] R. Rigelhof, Induced representations of locally compact groups, Acta. Math., 125 (1970), 155-187.

[Wod] M. Wodzicki, Excission in cyclic homology and in rational algebraic K-theory, Ann. Math., 129 (1989), 591-639.

Received October 25, 1996.

UNIVERSITETSPARKEN 5

DK-2100 KøBENHAVN $\varnothing$

DENMARK

E-mail address: gronbaek@math.ku.dk 